\title{
An integrated decision support system for ERP implementation in small and medium sized enterprises
}

\author{
Ying Xie \\ Lord Ashcroft International Business School, Anglia Ruskin University, Cambridge, UK, and \\ Colin Allen, Mahmood Ali \\ Business School, University of Greenwich, London, UK
}

\begin{abstract}
Purpose - Implementing ERP is a challenging task for small and medium sized enterprises (SMEs). This paper develops an integrated decision support system for ERP implementation (DSS_ERP) to facilitate resource allocations and risk analysis.

Design/methodology/approach - Analytical regression models are developed using data collected through a survey conducted on 400 SMEs that have implemented ERP systems, and are validated by a simulation model. The validated analytical regression models are used to construct a nonlinear programming model that generates solutions for resource allocations, such as time and budget.
\end{abstract}

Findings - ERP implementation cost increases along the time horizon, while performance level increases up to a point and remains unchanged. To maximise or achieve a certain level of performance within a budget limitation, CSFs are prioritised as: Project Management (highest), Top Management, IT, Users and Vendor Support (lowest). SMEs are recommended to concentrate effort and resources on CSFs that have a greater impact on achieving their desired goals while optimising utilisation of resources.

Research limitations/implications - DSS_ERP proves to be beneficial to SMEs in identifying required resources and allocating resources, but could be further tested in case studies for its practical use and benefits.

Practical implications - DSS_ERP serves as a useful tool for SMEs to predict required resources and allocate them prior to ERP implementation, which maximises the probability of achieving predetermined targets. It also enables SMEs to analyse risk caused by changes to resources during ERP implementation, and helps them to be better prepared for the risks.

Originality/value - The research contributes to the scarce research on ERP implementation using scientific methods. A novel nonlinear programming model is constructed for ERP implementation under time and budget limitations, facilitating resource allocations in an ERP implementation, which has not been reported in any previous research. The research offers a theoretical basis for empirical studies of resource allocations in ERP implementation. 
Key words: decision support system, ERP, critical success factor, nonlinear programming, simulation, SME

\section{Introduction}

Enterprise resource planning (ERP) systems automates core corporate activities and optimises the flow of information and resources throughout the entire supply chain (Umble et al., 2003). Small and medium sized enterprises (SMEs) have realised the usefulness and importance of this system, and prefer to adapt ERP systems to the business processes through customisation (Zach and Munkvold, 2012). SMEs have been found to be constrained by limited resources that are needed to address these issues, and are forced to compromise implementation and subsequently putting the success of ERP project at risk (Sun et al., 2005). As a result, ERP implementation becomes a real challenge for SMEs. In an ideal situation, SMEs would have implemented ERP successfully within limited budget and time duration. If there is a readily available and reliable tool to forecast efforts, schedules and costs required to achieve the desired success level in ERP implementation, SMEs will be able to plan ahead to acquire resources and increase the success rate of implementation. Since such a tool illuminates the relationships between the desired success level and the needed resources/resource allocation, it can provide proper justification for project planning. However, such a tool has not been reported in any of research on ERP, which is the driver of this research.

In general, when ERP implementation is considered, there are numerous parameters and factors affecting its success. Therefore, it will be difficult to develop a single precise mathematical model measuring the relationships among these factors. In this research, an integrated Decision Support System (DSS) for ERP implementation (DSS_ERP) is developed for SMEs, combining analytical regression models, a simulation model, and a nonlinear programming model, to predict ERP project implementation outcomes and facilitate allocating resources. This is different from the DSS for ERP software selection (Cebeci, 2009; Karsak and Ozogul, 2009), the DSS helping achieve enterprise wide integration of ERP systems across different departments in an organisation (Lea et al., 2005), and the DSS articulating the relationships between organisational profiles and ERP success (Rouhani and Ravasan, 2012); the DSS_ERP is a quantitative tool, linking Critical Success Factors (CSFs) to project outcomes measured by implementation cost, project duration and performance level, and exploring the impact of changes to budget limit and focus on individual CSFs. For the first time, a novel nonlinear programming model is constructed under time and budget constraints and built into DSS_ERP, facilitating decision makers in allocating resources (time spent on 
each CSF), and assisting in improving the progressing speeds in these CSFs, so that predetermined implementation goals can be achieved, such as maximising the performance level with the constraints on the implementation cost and project duration, or achieving a certain level of performance at the end of project duration. Compared with DSSs reviewed above, the DSS_ERP has three unique advantages: (1) it can act as an analytical tool to monitor ERP implementation progresses, (2) it facilitates decision making on resource allocations to achieve the predetermined targets and (3) it offers a risk analysis tool to analyse potential risk and opportunities caused by the changes.

The paper is organised as follows. A critique of literature is demonstrated in Section 2 to establish the need for the research. The research scope and methodology are discussed in Section 3. In Section 4, through a combination of empirical studies and modelling, DSS_ERP is developed incorporating analytical regression models, a simulation model and nonlinear programming models. Section 5 illustrates its application through Goal-Seeking and What-If analysis. Section 6 compares main findings with former research and demonstrates the contribution of the paper. Conclusions and future work are presented in Section 7.

\section{Literature review}

A comprehensive literature review is conducted by Schlichter and Kraemmergaard (2010), who classified research within ERP into eight research topics: implementation (30\%), managing ERP systems (20\%), optimisation of ERP (17\%), the ERP tool (14\%), ERP and supply chain management (7\%), ERP market and industry (7\%), education and training (3\%), and how to study ERP (1\%). The literature related to ERP implementation investigated how the ERP systems can be introduced into the organisation, including papers concerning ERP software selection, the various steps of implementation and related problems, CSFs, and business process reengineering during implementation.

The issues related to ERP implementation are classified as: Context, namely environmental context, organisational context and technological context (Raymond and Uwizeyemungu, 2007); Project Organisation, that is measured by project team competence, clear goals, project management and ERP selection (King and Burgess, 2006); Information Technology (IT) Infrastructure (Bernroider, 2008), including IT system readiness and data migration capability; and Support that is provided by top management, project champion and vendors (King and Burgess, 2006). There are numerous generic CSF based ERP implementation models that address key implementation issues and a wide range of CSFs are identified. Organisations are recommended to focus on them in order to improve the chances of successful implementation 
(Akkermans and van Helden, 2002). Holland and Light (1999) developed a CSF model for ERP implementation, which groups the CSFs into strategic and tactical factors. Each of the factors is specific to the ERP project. Somers and Nelson (2001) identified the top 10 CSFs ranked by US executives as follows: 1) Top management support; 2) Project team competence; 3) Interdepartmental co-operation; 4) Clear goals and objectives; 5) Project management; 6) Interdepartmental communication; 7) Management of expectations; 8) Project champion; 9) Vendor support; and 10) Careful package selection. Cantu (1999) defined a framework exclusively for SMEs based on five CSFs: Management/organisation, Process, Technology, Data and People. Each of the CSF is further analysed into a number of attributes reaching a total of 22 attributes. Although some authors break these CSFs into more detailed attributes while others define then in generic terms, regardless of which approach they take the CSFs themselves are generally similar and can be considered as such. The CSFs identified in these researches help the SMEs to better understand the impacts of the CSFs, however, the extent of these impacts are not clear and SMEs will not be able to make effective interventions in ERP implementations.

Schlichter and Kraemmergaard (2010) reported that case studies have been the most prevalent research methods in studying ERP with 32 per cent, followed by surveys accounting for 14 per cent, and only 9 per cent have used scientific methods. The limitations of case studies are that the conclusions obtained from the research are only applicable to the companies involved in the research work, and that the methodologies are not applicable to derive a generalised practical model to measure impacts of the CSFs on ERP implementation success level or to facilitate resource allocation to CSFs. In order to advance ERP related research and generalise the research outcomes, researchers have explored multiple research approaches that generate more robust, yet precise results (Cumbie et al., 2005). King and Burgress (2006) presented a new dynamic simulation model of ERP in order to better understand the relationship between CSFs and to encourage exploration of more appropriate implementation strategies. However this simulation model was developed qualitatively without any quantitative analysis. To address this problem, Parr and Shanks (2000) developed a project phase model (PPM) of ERP implementation projects which investigates the relationship between phases of ERP projects and CSFs. Using the two case studies, the PPM was claimed to be a useful model for ERP implementation and provides a template to suggest important CSFs during particular project phases. Rouhani and Ravasan (2012) developed an Artificial Neural Network (ANN) based expert system to imitate the relationship between organisational factors and ERP success factors, and to predict the probable success level. However, this model 
does not provide a quantitative means to measure success level, nor could it predict the numbers of resources needed to achieve a successful ERP implementation. For SMEs, focus on CSFs is important during the implementation process, but cost and project duration are very important factors for SMEs to consider prior to adopting an ERP system, due to limited resources and complex processes. Therefore, Plaza and Rohlf (2008) investigated the learning and performance of the project team, to find out how the training strategy can minimise the ERP project consulting cost and to provide an analytical method for predicting a project completion date. However, their work was limited to the use of analytical models to calculate project duration rather than resource allocation, and dynamic views are not given on the ERP implementation project processes. To synthesize the level of tangible and intangible costs required to achieve a given success level, Sun et al. (2005) developed a simulation model by converting CSFs into quantitative information, to help SMEs identify the key requirements (time spent on each CSF) and measurements (cost, schedule and goal achievement) that determine the achievement of ERP implementation. But this model does not have the functionalities of predicting resources needed for each CSF, and is of no use to SMEs in advanced planning. Furthermore, this model is developed from the data collected from six case studies and its robustness is not tested.

Former research on ERP implementation in SMEs reveals a need to develop a robust quantitative tool to assist ERP implementation in SMEs by identifying emphasis placed on CSFs, and resources allocated to each CSF. The tool should also demonstrate both the analytical and practical aspects of an ERP implementation, and offer a dynamic view of implementation process. Sun et al. (2005) proved that each CSF is associated with cost, schedule, and performance attributes that can be presented by mathematical functions, and that the dynamic ERP implementation environment can be quantified into CSFs and replicated in a simulation model. Through a non-empirical evaluation, Stensrud (2001) shortlisted regression analysis as the only parametric effort prediction system suitable for ERP projects. The regression analysis is able to express the relationship between a predictor variable (for example, budget and project duration) and the associated response variable (for example, ERP performance level) in mathematical form. However, due to the non-empirical nature of his research, there is no limitation on the context where this finding is applicable. Therefore, the purpose of this research is to develop a quantitative tool by combining three types of models: (1) ERP analytical regression models, in which the implementation cost, performance level and project duration are broken down by CSFs, and the relationships among them are obtained for 
each CSF quantitatively; (2) an ERP simulation model, which provides a dynamic view of ERP implementation process and verifies analytical models in (1); and (3) an ERP nonlinear programming model, which is constructed under time and budget limits, and generates solutions for the emphasis placed on CSFs, measured by the time and cost spent on those CSFs.

\section{Research scope and methodology}

\subsection{Critical Success Factors considered}

Cantu's framework is one of the prominent pieces of research investigating CSFs exclusively for ERP implementation in SMEs, and has been validated and widely considered in literature (Ho and Lin, 2004; Loh and Koh, 2004; Sun et al. 2005) as a guidance framework.

In order to develop a parametric effort prediction system for ERP implementation in SMEs, this research captures the predominant CSFs impacting on ERP implementation performance, and models them through regression analysis that creates expected values and provides confidence levels. To quantify the response variable, i.e., dynamic ERP implementation performance, into predictor variables, i.e., time and cost spent on CSFs, Cantu's framework is adopted in this research with some revisions.

To obtain experts' views on the CSFs recommended in Cantu's framework, and test the degree to which the CSFs are addressed during ERP implementation, in depth interviews were conducted with five ERP implementation consultants, two from the Information Technology sector, two from Manufacturing and one from Banking and Finance. In their opinions, none of the SMEs where they have worked with addressed all possible attributes listed in Cantu's framework, but all the SMEs value Top Management that assures commitment from top level, and Vendor Support that is required to establish the physical IT infrastructure and to gather information. Through the participations in three ERP implementation projects in SMEs (one manufacturing, and two retailers), the authors also observed that Users' attitude towards the new ERP system has a significant impact on the success rate of implementation. In addition, the competence of the project team plays a key role in making sure the ERP project is implemented within both budget and time duration.

Based on the information derived from multiple sources above, the authors consider the following five CSFs in this research:

- Top Management support (TM): the leadership, commitment and participation provided by the top management level to the project. It is part of CSF Management in Cantu's framework (Cantu, 1999), but regarded as the most important CSF by researchers (Somers and Nelson, 2001; Umble et al., 2003) and practitioners. It plays a 
critical role in reinforcing the degree of commitment of all employees to the implementation (Zhang et al., 2005;);

- Users: the level of participation by the end users during the implementation process, defined by users' perception, interest, IT skills, commitment and feedback on the overall ERP project (Nah et al., 2001; Zhang et al., 2005). Users are equivalent to People in Cantu's framework;

- IT infrastructure (IT): hardware and software readiness, presence of reliable IT architecture, the availability of databases with adequate quality and data migration readiness (Bajwa, et al., 2004). This CSF is the combination of Technology and Data in Cantu's framework.

- Project Management (PM): the on-going management of the implementation process to achieve specific project goals and objectives (Nah et al., 2001; Umble et al., 2003), including team competence, and the necessary business process reengineering although this rarely happens in SMEs. Project Management has gained its reputation as an important CSF (Somers and Nelson, 2001), and is considered in this research to replace Management (project team) and Process in Cantu's framework (Cantu, 1999).

- Vendor Support (VS): the application of external expertise in providing training, technical knowledge, maintenance, back up support, emergency management, updates, service responsiveness and reliability (Zhang et al., 2005; Remus, 2007). Vendor Support is not considered in Cantu's framework (1999), but is particularly important for SMEs since they lack the experience and skills necessary to grasp all the complexities of implementing ERP system (Markus and Tanis, 2000).

\subsection{Deductive research approach: regression curves adopted}

Complementing mathematical modelling with an empirical survey, this research uses both the linear curve and the exponential curve as means to represent the implementation cost and performance level over project duration, respectively. The implementation cost is the cumulative cost of the overall ERP implementation project. The performance level is defined as the percentage of the organisation's target functional requirement met by the ERP implementation. The project duration is defined the time elapsed from the initial training phase to the final go live phase, covering the configuration, testing, and conversion phases that are common to various ERP system adoption models (Parr and Shanks, 2000). Although it is argued that ERP implementation is a never-ending cycle of continuous improvement, the scope 
of this research is limited to ERP implementation issues after package selection and project planning. The focus of the research is on the efforts put into ERP implementation from the initial training until immediate success is achieved. The S-Curve is a well-known project management tool and it is defined as "a display of cumulative costs, labour hours or other quantities plotted against time” (Project Management Institute Standards Committee, 2000). The common characteristics of an ERP implementation project demonstrate that implementation progress grows slowly in the initial training phase, then grows rapidly in configuration/testing/conversion stage, and in turn reaches an asymptotic maximum when the project goes live (Cioffi, 2005), as shown in Fig. 1, where the progress is measured as the contribution to performance level. Fig. 1 is a simplified version of the S-Curve when the startup effect in the project planning stage is not considered, and is called an exponential curve which is robust and has been widely applied to predict and model the impact of competence on performance (Plaza and Rohlf, 2008). When the initial planning stage and final phasing off stage are not considered, the relationship between cost and time can be assumed linear, and has been adopted as such in the project management literature (Fulkerson 1961; Babu and Suresh, 1996). Thus, a linear curve is adopted to model the relationship between implementation cost and project duration. The methodology described above is usually called a deductive research approach, beginning with general theories or models and narrowing them down to the research interest.



Fig. 1 An exponential curve for ERP implementation project

\subsection{Inductive research approach: development of models based on survey data}

Inductive research approaches are also adopted in this research, beginning with specific observations and measures drawn from SMEs who have completed at least one ERP 
implementation, empirically evaluating implementation cost, performance level and project duration broken down by CSFs, and obtaining two curves for each CSF: 1) a Cost vs Time linear curve showing cost expended over time spent on a CSF, and 2) a Progress vs Time exponential curve expressing the percentage of performance level contributed by a CSF along the time horizon. These curves are formulated by the analytical models, which make it possible to predict the impact of attention on CSFs and to accurately calculate performance level, implementation cost and project duration that are not available in the literature.

The European Commission defines SMEs using three broad parameters: micro enterprises are companies with up to 10 employees, small enterprises employ up to 50 workers, and medium-sized enterprises have more than 50 but less than 250 employees. Findings from Buonanno et al. (2005) clearly show that the rate of ERP system adoption is quite low among both micro (3\%) and small enterprises (12\%), but higher in medium sized enterprises (47\%). Significant differences are found to exist between small enterprises and medium enterprises regarding the objectives and constraints of ERP adoption and implementation (Laukkanen, et al., 2007). One of the constraints is the resources available to ERP implementation (including budget, time, number of staff, their knowledge about ERP system, etc).

In order to reflect a realistic implementation, a representative sample needs to be chosen to collect information and construct the analytical regression models. A sample of SMEs is defined with the following criteria:

Criterion 1: $\quad$ The SMEs are medium-sized enterprises, having 50-150 staff,

Criterion 2: $\quad$ The SMEs have completed at least one ERP project

Criterion 3: During ERP implementation, the SMEs have addressed the CSFs in Section 3.1

Having Criterion 1 met, the medium-sized enterprises with a higher rate of ERP adoption are chosen, and we can assume that the discrepancies in staff allocations to each CSF in the surveyed SMEs are insignificant, so the ERP implementations in the surveyed organisations can be analysed using the same regression curves. The parameters of the analytical models are obtained using least square methods. To ensure the mathematical modelling reflects a realistic ERP implementation, the results obtained from the analytical models are verified and validated by Monte Carlo simulation (Balakrishnan et al., 2007).

Having Criterion 2 and 3 met, the information which is going to be used to make the prediction, i.e., time spent on each CSF considered in this research, and the information which is to be predicted, i.e., implementation cost and performance level, can be obtained from the sample. 
Taking into account that SMEs are constrained by time and budget limitations, this research develops DSS_ERP, facilitating resource allocations to achieve the predetermined goals. Comparison of the exponential curves for CSFs reveals the progress made by them over time, and their impacts on the overall project progress are analysed. The CSFs that make the major contribution to the ERP project are identified and addressed with greater focus. The DSS_ERP developed involves real-life data and information, and can demonstrate both the analytical and practical aspects of an ERP implementation. It offers a guidance tool for SMEs to predict project outcomes and evaluate implementation strategies prior to ERP implementation. It can also facilitate decision makers in analysing the impacts of changes to resource allocations on the overall ERP implementation performance.

\section{The proposed decision support system}

The DSS facilitates the decision making process by compiling raw data into useful information that decision makers can effectively use and apply to organisational and business decisions. There is a need to develop a DSS_ERP to determine what inputs are required to reach specific goals, such as performance level, project duration and implementation cost, which is known as Goal-Seeking analysis. Based on the results obtained from Goal-Seeking analysis, the ERP implementation strategies can be developed and evaluated. In addition, DSS_ERP can be used to conduct What-If analysis, i.e., to determine the impact of the possible changes, such as tuning focus on some CSFs, or increasing budget limitation, on the overall project performance.

The procedure for developing DSS_ERP is outlined in Fig.2, incorporating three types of models:

(a) ERP analytical regression models where ERP project performance level is calculated according to initial resources allocation on the CSFs,

(b) ERP simulation model providing techniques to validate the analytical models developed in (a) and help develop a more rigorous theory of ERP implementation,

(c) ERP nonlinear programming model that is constructed to conduct Goal-Seeking and What-If analysis, i.e., obtain the solutions for the predetermined goals and analyse the impacts of changes to ERP performance. 


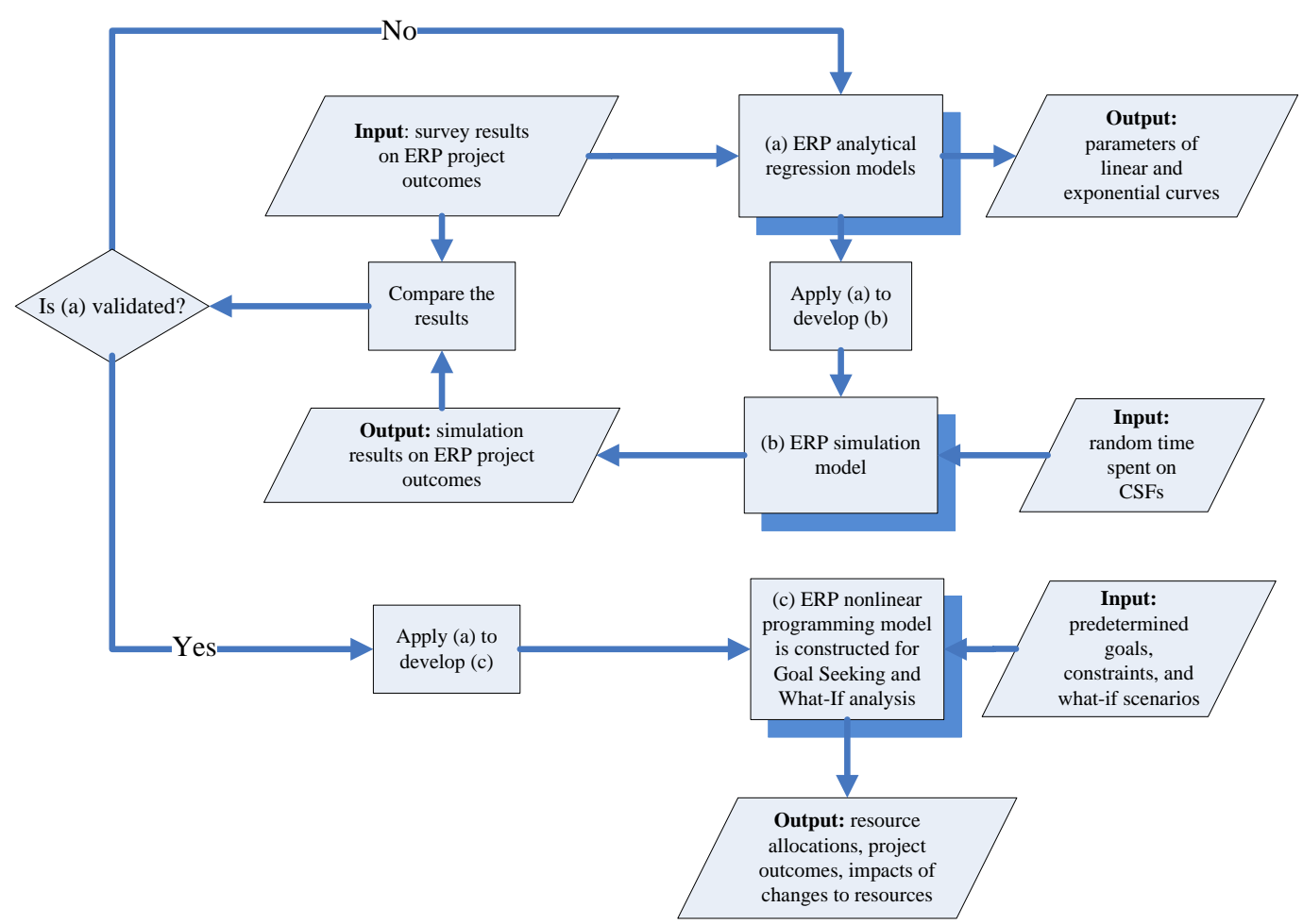

Fig.2 development and structure of DSS_ERP

The stepwise representation of the integrated DSS_ERP is given below.

Step 1. Using data collected from the survey as an input, i.e., time spent on each CSF as independent variables and cost incurred or progress level achieved at CSF as dependent variables, calculate the unknown parameters of linear and exponential curves, and construct analytical regression models (a).

Step 2. Having time spent on each CSF as inputs, develop a Monte Carlo simulation model where cost and progress level attributed to each CSF are calculated using models (a), and average implementation cost and performance level are obtained after a number of replications. The average project outcomes are compared with the average original survey results to verify the validity of models (a).

Step 3. If models (a) are not validated, Step 1 is repeated to develop new models (a) by choosing different types of regression curves. If models (a) are validated, they are applied to construct a nonlinear programming model (c) under time and budget constraints. The nonlinear programming model is used to conduct Goal Seeking analysis that facilitates resource allocations to achieve predetermined goals, and What-If analysis that analyse the impacts of changes to resources allocated.

The three types of models are integrated in such a way that nonlinear programming models can only be constructed when analytical regression models are verified to be valid by a 
simulation model. On the other hand, a simulation model can only be developed when the relationships between cost and time, and progress and time are determined by analytical regression models.

\subsection{Step 1. ERP analytical regression models}

Based on the authors' observations and research on ERP implementations (Sun et al., 2005), the total cost of ERP implementation increases along with the total time spent, however, the overall performance or success rate increases up to a certain point then remains unchanged, following the exponential curve shown in Fig.1. These relationships are attributed to the contributions made by each CSF. More cost is incurred if a longer time period is spent to address a CSF, but the progress of a team who work on a CSF follows an exponential curve, as demonstrated in Plaza and Rohlf (2008) for a project management team. It is observed that the progresses made by other CSFs, such as top management, end users, IT infrastructure and vendor support also follow the exponential curve. Inside the company, the teams who address these CSFs lack both knowledge of and experience with the system they are about to implement. Outside the company, the supporting team from the ERP vendors lack knowledge and experience about their customer (the company implementing ERP). Therefore, the initial contribution made by a CSF team to the ERP implementation performance level is low, but increases up to a performance threshold sometime during the progression of the project.

At CSF level, the relationship between time and cost is represented by the Cost vs Time linear curve, and is modelled by a linear regression model (Fox, 2008) in formula (1):

$\operatorname{cost}_{i}\left(t_{i}\right)=d_{i} \cdot t_{i}$

Where $d_{i}$ is the coefficient of the cost function and $t_{i}$ represents time spent on the $C S F_{i}$, which is one of CSFs addressed in the ERP implementation. A constant is omitted in formula (1) as, although some costs may be incurred when no time is spent, those costs are so low relative to the costs incurred in spending time that they can effectively be regarded as zero, i.e., $\operatorname{cost}_{i}\left(t_{i}\right)=0$ when $t_{i}=0$.

The implementation cost of ERP is obtained as:

$\operatorname{Cost}\left(t_{1}, t_{2}, \ldots, t_{M}\right)=\sum_{i=1}^{M} \operatorname{cost}_{i}\left(t_{i}\right)$

Where $M$ denotes the total number of CSF considered.

A Progress vs Time exponential curve is used to express the relationship between the progress made by a CSF against time, and formulated as the exponential regression model (Fox, 2008) in formula (3). The progress, denoted $P F_{i}$, is measured as the percentage of the 
performance level contributed by $C S F_{i}$, and reaches the performance threshold level $p_{i}$ when unlimited time (associated with unlimited cost) is spent on it, i.e., $P F_{i}\left(t_{i}\right)=p_{i}$ when $t_{i}=\infty$. $P F_{i}\left(t_{i}\right)=p_{i} \cdot\left(1-e^{-k_{i} t_{i}}\right)$

Where $p_{i}$ denotes the performance threshold of $C S F_{i}$, and $k_{i}$ is the progressing coefficient. The progressing coefficient $k_{i}$ directly relates to the rate of progress made by a team; however, since the ERP project team is diverse by nature, and will vary considerably with the context within which ERP is implemented (Raymond and Uwizeyemungu, 2007), $k_{i}$ is difficult to calculate accurately. To obtain a single value of $k_{i}$ that represent the changes in performance of the team, and enhance the accuracy of $k_{i}$, the SMEs chosen for the survey are required to meet Criteria 1-3 in Section 3.3.

The performance level of ERP implementation $P F$ is calculated as:

$P F\left(t_{1}, t_{2}, \ldots, t_{M}\right)=\sum_{i=1}^{M} P F_{i}\left(t_{i}\right)$

Having the surveyed results as inputs, the parameters $d_{i}, p_{i}$ and $k_{i}$ are the outputs to the analytical regression models, and are calculated using the least square method which finds the best fit Cost vs Time linear curves and Progress vs Time exponential curves for the observed data. The coefficient of determination of the regression curve for $C S F_{i}$, denoted $R_{i}^{2}$, is calculated to describe how well the regression curve fits the original set of data. If the average

value of $R_{i}^{2}$ is lower than 0.5 , i.e., $\frac{\sum_{i=1}^{M} R_{i}^{2}}{M}<0.5$, other regression curves need to be experimented with and compared to the observed data until the average value of $R_{i}^{2}$ is higher than 0.5 .

\subsection{Step 2. ERP simulation model}

A Monte Carlo simulation model is developed to verify the validity and effectiveness of the analytical regression models developed in Section 4.1, in such a way that outputs from the simulation model are compared with the observed ERP implementation performances to see if the regression models work as expected.

Due to limited resources, the majority of SMEs implement ERP using a sequential approach (Sun et al., 2005), addressing CSFs sequentially rather than simultaneously. The simulation model is thus constructed as a time dependent sequential model. The input data to the simulation model are: 1) time spent on each CSF, and 2) number of replications. Time spent on each CSF is a random independent variable to the simulation model, and the probability distribution is established for it by examining the historical outcomes, i.e., dividing the frequency of each observation by the total number of observations using formula (5). 
$\operatorname{prob}\left(t_{i}^{\prime}\right)=\frac{n_{t_{i}^{\prime}}}{\sum_{i=1}^{N} n_{t_{i}^{\prime}}}$

Where $t_{i}^{\prime}$ is a possible value that $t_{i}$ takes, $N$ denotes the total number of possible values of $t_{i}$, and $n_{t_{i}^{\prime}}$ is the frequency of $t_{i}^{\prime}$ or the number of times $t_{t_{i}^{\prime}}$ occurs.

In each replication, random numbers are used to simulate values for time $t_{i}$ from the probability distribution in (5) and these values are substituted to (1) and (3) to obtain cost and progress for each CSF, and ultimately the total cost and total achievement of the overall ERP implementation.

The above process is repeated for a number of replications, and the following outputs are generated from the simulation model: 1) average cost spent and progress made by CSF; and 2) three average project outcomes measures, i.e., project duration, implementation cost and performance level for the overall ERP implementation.

The outputs from the simulation model are independent random variables, each with finite mean and variance. According to the Central Limit Theorem (Weiss, 2012), for a relatively large sample size (usually 30 or more), the possible samples means of the simulation outputs are approximately normally distributed. The sample means are compared with the observed ERP implementation in terms of the three average project outcomes. If the observed results are within $99 \%$ confidence intervals of the sample means, the regression models are verified and resemble the ERP implementation in reality. Otherwise, the models need to be modified, which means either the parameters need to be recalculated or other types of regression models are selected. However, in this research, after experimentation with all types of regression models, linear and exponential regression models are found to be the ones that best express the relationships between time and cost, and time and progress. Therefore, the adjustment lies in parameters in these models.

\subsection{Step 3. ERP nonlinear programming model}

A nonlinear programming model (Taha, 2011) is developed to optimise ERP implementation to achieve the predetermined goals which are expressed in mathematical manner, and are subject to a number of constraints on cost, project duration, and vendor support level. If the goal is to maximise the overall performance level of ERP implementation, the objective function can be formulated as:

$\operatorname{Max} \quad P F\left(t_{1}, t_{2}, \ldots, t_{M}\right)=\sum_{i=1}^{M} P F_{i}\left(t_{i}\right)$

s.t. $\quad \sum_{i=1}^{M} t_{i} \leq T$ 


$$
\begin{aligned}
& \sum_{i=1}^{M} \operatorname{cost}_{i}\left(t_{i}\right) \leq C \\
& t_{i} \geq 0 \\
& t_{i} \leq T_{i}
\end{aligned}
$$

Where $T$ and $C$ are the limitations on the overall time and cost to be spent the project, and $T_{i}$ is the constraint on the time spent to address $C S F_{i}$. If a different goal is set up with different constraints, the formulae (6) and (7) need to be changed accordingly and this will be illustrated through an application in Section 5. The constrained nonlinear programming model in (6-7) cannot be solved explicitly for symbolic solutions, but a wide range of optimisation tools such as Excel's Solver and CPlex can be used to solve it numerically when the parameter values are given. The algorithms implemented by the optimisation tools vary with the solvers adopted, and Excel's Solver uses the Generalised Reduced Gradient (GRG) method, which is a generalisation of the Steepest Ascent (or Steepest Descent) method (Taha, 2011).

\section{Illustrative examples}

\subsection{Survey results}

Using random sampling, from January to April 2011, an Internet based survey was conducted on 400 SMEs in the UK and North America, these countries having the highest concentration of SMEs that have implemented an ERP project. The population was selected through ERP vendors' websites, Thomson Data, SAP users groups and small business association websites. SMEs were requested only to complete the survey if they met all of the selection Criteria 1-3 in Section 2. By the end of the survey, 80 responses were received but only 60 were valid responses after excluding incomplete, inconsistent (e.g., the total performance level is not the sum of the performance levels contributed by the CSFs), and incorrect (e.g., 20\% performance level is achieved without any cost incurred) responses. The response rate is calculated to be $15 \%$ which is considered reasonable compared with the response rates achieved in other research. The activity categories of SMEs which participated in the survey and provided valid responses encompass Information Technology (15\%), Manufacturing (28\%), Banking and Finance (10\%), Education (2\%), Telecommunication (14\%), Utility (9\%) and others (23\%). Surveying SMEs in different locations (UK and USA) and industries ensures that the model developed is relatively more generalisable.

In Table 1, the mean values of project duration, implementation cost and performance level achieved by the 60 SMEs are shown in the last column, these being the sum of the average time, cost and progress broken down by the five CSFs, as presented in columns 2-6. 
Table 1: Mean values of ERP implementation outcomes in the surveyed SMEs

\begin{tabular}{lllllll}
\hline & $\boldsymbol{C S F}_{\mathbf{1}}-\mathbf{T M}$ & $\begin{array}{l}\boldsymbol{C S F}_{\mathbf{2}} \\
\text { Users }\end{array}$ & $-\boldsymbol{C S F}_{\mathbf{3}}-\mathbf{P M}$ & $\boldsymbol{C S F}_{\mathbf{4}}$-IT & $\boldsymbol{C S F}_{\mathbf{5}}-\mathrm{VS}$ & Total \\
\hline Time (Days) & 13 & 29 & 30 & 36 & 19 & 127 \\
Cost (dollars) & 8,894 & 26,187 & 27,030 & 44,178 & 25,293 & 131,582 \\
Performance level (\%) & 8 & 16 & 16 & 15 & 10 & 64 \\
\hline
\end{tabular}

\subsection{Analytical regression models for the observed data}

At the CSF level, the data points plotted in Fig. 3 from the observed data show the accumulated cost and contribution to performance level as a function of time and, using Step 1, the smooth regression curves are the least square fitted Cost vs Time linear curves and Progress vs Time exponential curves. Employing formula (1) and (3), the values of $d_{i}, k_{i}$ and $p_{i}$ are obtained using least square methods and presented in Table $2 . R_{i}^{2}$ is given in Table 2 and Fig. 3 to describe how well the regression curves fits the original set of data. The average value of $R_{i}^{2}$ for Cost vs Time curve is 0.75 , and the average value for Progress vs Time curve is 0.77 , indicating that the selected regression curves are an acceptable fit for the observed data.

Table 2: Values of $d_{i}, k_{i}, p_{i}$ and $R_{i}^{2}$ for $\mathrm{CSF}_{\mathrm{i}}$

\begin{tabular}{cccccc}
\hline Parameters & $\boldsymbol{C S F}_{\mathbf{1}}$-TM & $\boldsymbol{C S F}_{\mathbf{2}}$-Users & $\boldsymbol{C S}_{\mathbf{3}}$-PM & $\boldsymbol{C S F}_{\mathbf{4}}$-IT & $\boldsymbol{C S F}_{\mathbf{5}}$-VS \\
\hline $\boldsymbol{d}_{\boldsymbol{i}}$ & 659.92 & 656.28 & 719.66 & 1361 & 1770.7 \\
$\boldsymbol{R}_{\boldsymbol{i}}^{2}$ - Cost curve & $\mathbf{0 . 9 1}$ & $\mathbf{0 . 6 1}$ & $\mathbf{0 . 6 8}$ & $\mathbf{0 . 7 7}$ & $\mathbf{0 . 7 9}$ \\
$\boldsymbol{k}_{\boldsymbol{i}}$ & 0.045 & 0.163 & 0.040 & 0.076 & 0.143 \\
$\boldsymbol{p}_{\boldsymbol{i}}$ & 19.03 & 17.13 & 24.26 & 19.28 & 12.94 \\
$\boldsymbol{R}_{\boldsymbol{i}}^{\boldsymbol{2}}$-Progress curve & $\mathbf{0 . 9 8}$ & $\mathbf{0 . 6 1}$ & $\mathbf{0 . 8 3}$ & $\mathbf{0 . 7 7}$ & $\mathbf{0 . 6 6}$ \\
\hline
\end{tabular}

For each CSF, the Cost vs Time linear curve and Progress vs Time exponential curve are formulated as follows:

$$
\begin{aligned}
& \text { CSF }_{1} \text {-TM: } \quad \operatorname{cost}_{1}\left(t_{1}\right)=659.92 \cdot t_{1} \\
& P F_{1}\left(t_{1}\right)=19.03 \cdot\left(1-e^{-0.045 \cdot t_{1}}\right) \\
& \mathrm{CSF}_{2} \text {-Users: } \quad \operatorname{cost}_{2}\left(t_{2}\right)=656.28 \cdot t_{2} \\
& P F_{2}\left(t_{2}\right)=17.13 \cdot\left(1-e^{-0.163 \cdot t_{2}}\right) \\
& \operatorname{CSF}_{3} \text {-PM: } \quad \operatorname{cost}_{3}\left(t_{3}\right)=719.66 \cdot t_{3} \\
& P F_{3}\left(t_{3}\right)=24.26 \cdot\left(1-e^{-0.04 \cdot t_{3}}\right) \\
& \text { CSF }_{4} \text {-IT: } \quad \operatorname{cost}_{4}\left(t_{4}\right)=1361 \cdot t_{4} \\
& P F_{4}\left(t_{4}\right)=19.28 \cdot\left(1-e^{-0.076 \cdot t_{4}}\right) \\
& \operatorname{CSF}_{5} \text {-VS: } \quad \operatorname{cost}_{5}\left(t_{5}\right)=1770.7 \cdot t_{5} \\
& P F_{5}\left(t_{5}\right)=12.94 \cdot\left(1-e^{-0.143 \cdot t_{5}}\right)
\end{aligned}
$$



CSF1 TM
curves curves

CSF3 PM curves

CSF4 IT curves
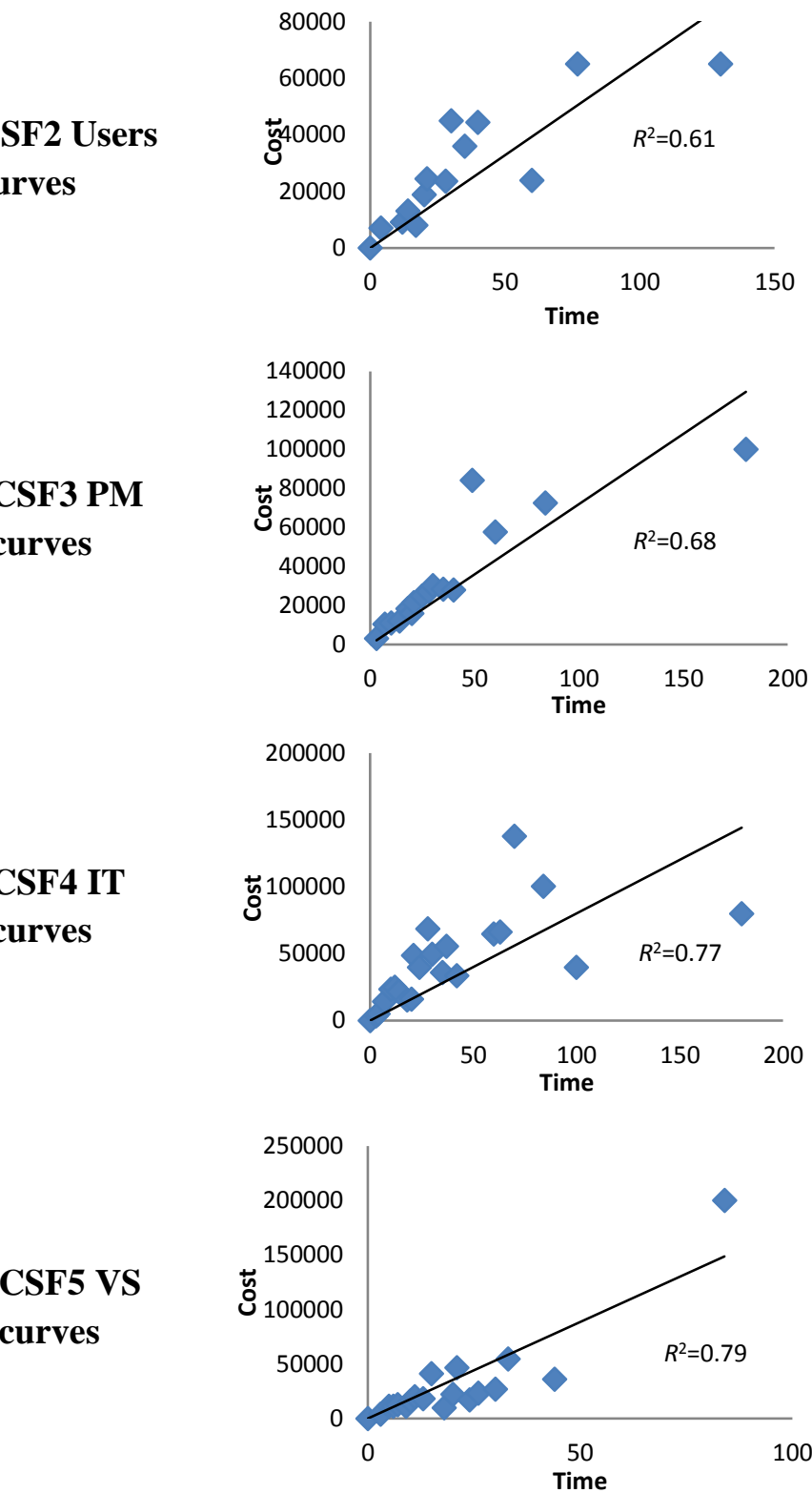
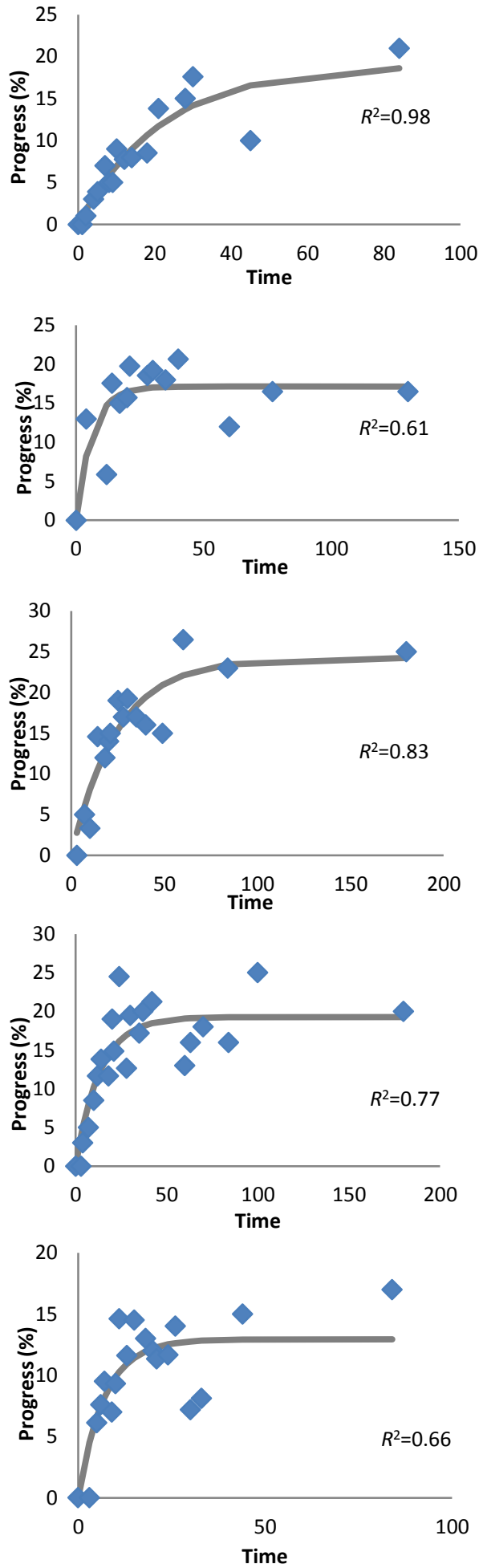

Fig. 3 Cost vs Time and Progress vs Time curves for CSFs

\subsection{Verification of the models}

The validity and effectiveness of analytical models in formulae (8-17) need to be verified before they are applied to develop DSS_ERP. Following Step 2, Verification is conducted by comparing the results from the analytical models with observed implementation performance. 
Time spent on $C S F_{i}$, noted as $t_{i}$, acts as the random input to the simulation model, and its probability distribution is calculated using formula (5). The probability distributions of $t_{i}$ are presented in Appendix A. Due to the fact that 60 valid responses are used to develop the analytical regression models, the average project outcomes for the overall ERP implementation are obtained by repeating the simulation 60 times in order to imitate reality. Taking a sample of 30 simulation outputs, the $99 \%$ confidence intervals of the sample means are compared to the observed data in Table 3. The observed data fall within the $99 \%$ confidence interval values of the sample means, verifying that the analytical models in (8-17) closely resemble the performance of the CSFs in reality, and work as expected.

Table 3: A comparison of the observed results and simulated results

\begin{tabular}{lccc}
\hline & Project duration & Implementation cost & Performance level (\%) \\
\hline $\begin{array}{l}\text { Observed results } \\
\text { 99\% confidence interval of } \\
\text { simulation results }\end{array}$ & 127 & 131,582 & 64 \\
\hline
\end{tabular}

Having verified the analytical models, the values of $d_{i}, k_{i}$ and $p_{i}$ in Table 2 reflect realistic relationships between cost and time, and progress and time for $C S F_{i}$, and reveal the following features:

- Comparing the values of $d_{i}$, VS and IT are much more costly than the other CSFs, which indicate that knowledge transfer from the external consultants and purchase of software and hardware systems are expensive components of the overall ERP implementation. This feature is consistent with the findings in Sun et al. (2005) and Plaza and Rohlf (2008).

- Comparing the values of $k_{i}$, Users and VS have higher progressing speeds than the others. The surveyed SMEs provided different levels of education and training to the end users before, during and after ERP implementation; this training helps the users to understand how the ERP system works and realise the full benefits of it. Compared with other CSFs, the users learn and progress faster as they only use one or two modules of the ERP system, such as financial management or inventory management. The VS is offered externally by the vendors, who have a greater knowledge of the ERP system, so it progresses more quickly than the other CSFs; however, it takes time for the vendors to understand and work out how the ERP system will meet the customer's functional requirements. This means Users and VS progress quicker in making contributions to performance level and need less resources.

- With respect to the values of $p_{i}, \mathrm{PM}, \mathrm{TM}$, and IT make greater contributions to the performance level than Users and VS. This means excellent project management, 
effective top management and integrated IT system, and should be the subject of intense focus. This includes defining clear objectives, having a competitive project team, developing clear work and resource plans, setting up hardware and software system and getting support from top management for ERP implementation.

\subsection{Goal-Seeking analysis}

Once the analytical models are verified using the simulation model, DSS_ERP are developed by constructing nonlinear programming objective functions to achieve predefined goals, as set out in Step 3. Goal Seeking analysis is conducted to make decisions on the following variables:

- $t_{i}$, time needed to address $C S F_{i}$

- $k_{i}$, progress coefficient of $C S F_{i}$

By setting up the goals, DSS_ERP calculates either or both $t_{i}$ or $k_{i}$, which in turn can help decision makers to focus efforts and resources on CSFs that have a greater impact on achieving their desired goals, and to develop corresponding implementation strategies. Three goals are established with constraints:

Goal 1: $\quad$ with a project duration of less than or equal to 180 days, and a budget limit of 100,000 dollars, determine the time to be spent on each CSF so that the performance level is maximised.

The nonlinear programming formulation for Goal 1 would be written as:

$\operatorname{Max} P F\left(t_{1}, t_{2}, t_{3}, t_{4}, t_{5}\right)$

s.t. $\quad \sum_{i=1}^{M} t_{i} \leq 180$

$$
\begin{aligned}
& \sum_{i=1}^{M} \operatorname{cost}_{i}\left(t_{i}\right) \leq 100000 \\
& t_{i} \geq 0
\end{aligned}
$$

Using formula (4), the objective function is rewritten as:

$$
P F\left(t_{1}, t_{2}, t_{3}, t_{4}, t_{5}\right)=P F_{1}\left(t_{1}\right)+P F_{2}\left(t_{2}\right)+P F_{3}\left(t_{3}\right)+P F_{4}\left(t_{4}\right)+P F_{5}\left(t_{5}\right)
$$

Substituting (8-17) to (18), the objective function becomes:

$$
\begin{aligned}
& \operatorname{Max} P F\left(t_{1}, t_{2}, t_{3}, t_{4}, t_{5}\right) \\
= & 19.03 \cdot\left(1-e^{-0.045 \cdot t_{1}}\right)+17.13 \cdot\left(1-e^{-0.163 \cdot t_{2}}\right)+24.26 \cdot\left(1-e^{-0.04 \cdot t_{3}}\right)+ \\
& 19.28 \cdot\left(1-e^{-0.076 \cdot t_{4}}\right)+12.94 \cdot\left(1-e^{-0.143 \cdot t_{5}}\right)
\end{aligned}
$$

The above nonlinear programming model is solved using Excel's Solver, which uses the GRG procedure. The solutions of $t_{i}$ and resultant project outcomes are listed in Table 4. Without 
extra external consulting, training or staff allocation, the progressing coefficients $k_{i}$ are kept with the same values as Table 2, and the maximum performance level achieved is $73.276 \%$, with the project duration being 115 days, and more time allocated to PM and TM. This is attributed to their higher performance thresholds $p_{i}$ and slower progressing speeds $k_{i}$, and they are prioritised and given greater focus. However, it should be noted there is less time allocated to IT, as it is more expensive to address, but it more rapidly makes contributions to performance level. When an SME aims to maximise performance level within a budget limitation, increased focus is given to the CSFs that can make greater contributions to the performance level, while maintaining lower cost. It is worth pointing out that, during rapid implementation, the budget available does not allow the CSFs with higher performance thresholds and slower progression speeds, for example, PM and TM, to reach the performance thresholds, so CSFs that progress more rapidly should be given greater focus so that a higher performance level can be achieved within both budget and time limitation. However, this analysis is outside the research scope of this paper, and will be discussed in the future work.

If the SME aims to achieve a higher performance level at the end of the project without incurring more cost as a function of time, the progressing speeds of the teams who address CSFs need to be increased. To achieve this, additional resources need to be allocated, including more commitment from top management, more advanced IT systems, more staff training, increased size of the project team, higher level of external support etc. As a result of adding extra resources, the overall implementation cost is increased. To analyse the impacts of a higher level of target on the progressing coefficients $k_{i}$, and simplify the mathematical modelling, the extra resource cost is excluded from the budget limitation. This imposes a tighter cost constraint on the solutions, and gives more room to the decision makers to develop the corresponding implementation strategy.

The decision makers can choose one or multiple CSFs to make changes, depending on the availability of resources. The majority of SMEs cannot afford to upgrade to more advanced IT systems or to remove full time staff from their everyday duties to support ERP implementation, so more commitment from top management, additional time for the project, more training for users and the project team, and extra consulting support are more appropriate strategies. Using the parameters obtained from goal 1, the decision maker set up goal 2 as follows:

Goal 2: $\quad$ with a budget limit of 100,000 dollars, and a project duration of 115 days, determine the time to be spent on each CSF, and the progressing coefficients of 
PM and TM, so that the performance level is at least $75 \%$ at the end of the project.

Goal 2 is formulated as follows, taking into account the parameters obtained in Goal 1:

$$
P F\left(t_{1}, t_{2}, t_{3}, t_{4}, t_{5}, k_{1}, k_{3}\right) \geq 75 \%
$$

$$
\begin{array}{ll}
\text { s.t. } & \sum_{i=1}^{M} t_{i} \leq 115 \\
& \sum_{i=1}^{M} \operatorname{cost}_{i}\left(t_{i}\right) \leq 100000 \\
& t_{i} \geq 0 \\
& k_{1} \geq 0.045 \\
& k_{3} \geq 0.040
\end{array}
$$

The solutions of $t_{i}, k_{1}$ and $k_{3}$ are depicted in Table 4 , suggesting more resources are allocated to TM and PM, in order to increase the progressing speeds up to 0.050 and 0.046 , respectively. The increments of progressing speeds are calculated as: $(0.050-0.045) / 0.045=11 \%$ for TM and (0.046-0.04)/0.046=15\% for PM, and are thought to be achievable. The time spent on the CSFs remains the same as the solutions for goal 1.

If the decision maker decides to provide more user training and purchase additional external consultancy to achieve the $75 \%$ performance level at the end of project duration, goal 3 would be setup:

Goal 3: $\quad$ with a budget limit of 100,000 dollars, and a project duration of 115 days, determine the time to be spent on each CSF, and the progressing coefficients of Users and VS, so that the performance level is at least $75 \%$ at the end of the project.

Goal 3 is formulated in the same way as goal 2, but the decision variables and constraints are changed:

$$
P F\left(t_{1}, t_{2}, t_{3}, t_{4}, t_{5}, k_{2}, k_{5}\right) \geq 75 \%
$$

s.t.

$$
\begin{aligned}
& \sum_{i=1}^{M} t_{i} \leq 115 \\
& \sum_{i=1}^{M} \operatorname{cost}_{i}\left(t_{i}\right) \leq 10000 \\
& t_{i} \geq 0 \\
& k_{2} \geq 0.163 \\
& k_{5} \geq 0.143
\end{aligned}
$$

As shown in Table 4, the time spent on the CSFs is identical to the solutions for goals 1 and 2, but the progressing speeds of Users and VS are increased to 0.199 and 0.189 respectively. 
Compared with the solutions for goal 2, the increments of progressing speeds are much higher, i.e., $22 \%$ for Users and $32 \%$ for VS, and are more difficult to achieve. This can be explained as being due to the performance thresholds of Users and VS being smaller than the performance thresholds of TM and PM, and the time spent on Users and VS being shorter than TM and PM, so the same level of increment in progressing speeds of Users or VS results in a smaller increment in contributions to the performance level of ERP implementation.

\subsection{What-If analysis}

The goal seeking analysis in Section $\mathbf{5 . 4}$ acts as a useful guidance tool for decision makers in developing implementation strategies and allocating resources for ERP implementation, but it is noted that the solutions for each goal are constrained by the limitations on the project duration and implementation cost. What if changes are made to these constraints? During the planning phase of an ERP project, project duration and budget have to be estimated, but they will change during implementation if extra resources become available, for example extra funds, or current resources become unavailable, for example the resignation of the project manager or withdrawal of vendor support. The decision makers will be more prepared for the potential risk and opportunities resulting from the changes if they have a prior understanding of the impacts caused by the changes.

Using goal 1 as Scenario 0, a What-If analysis is conducted to explore scenarios when changes are suggested, and seven other scenarios are defined. Scenarios 1-4 explore the impact of changes to the budget limitation, and Scenarios 5-7 analyse the impact of tuning focus on the CSFs, measured by the budget spent on the CSFs.

Scenario 1: with the budget limit increased by 5\% to 105,000 dollars, and no limit on project duration, determine the time to be spent on each CSF to maximise the performance level achieved at the end of the project.

Scenario 2: the budget limit is increased by $20 \%$ to 120,000 dollars, and other constrains remain as in Scenario 1.

Scenario 3: the budget limit is increased by 200\% to 300,000 dollars, and other constrains remain as in Scenario 1.

Scenario 4: the budget limit is increased by 300\% to 400,000 dollars, and other constrains remain as in Scenario 1.

Scenario 5: with the project duration less than or equal to 180 days, no vendor support, and a budget limit of 100,000 dollars, determine the time to be spent on each CSF so that the performance level is maximised. 
Scenario 6: with an additional 5\% on the PM budget, the project duration less than or equal to 180 days, and a total budget limit of 105,000 dollars, determine the time to be spent on each CSF so that the performance level is maximised.

Scenario 7: with an additional 5\% on the Users budget, the project duration less than or equal to 180 days, and a total budget limit of 105,000 dollars, determine the time to be spent on each CSF so that the performance level is maximised.

The objective functions in these scenarios are identical to formula (18), but with different constraints. The results for these scenarios are obtained using Excel's Solver and presented in Table 5 and Fig. 4. $\Delta C$ is the change in $C$ - the limitation in cost, calculated as the percentage of the difference between $C$ setup in each scenario and $C$ in the Scenario 0. $\triangle P D$ and $\triangle P F$ are the changes caused in project duration and performance level, and are calculated in the same way.

As shown in Fig.4, the results from Scenarios 1-4 indicate that $\Delta C$ has a much stronger impact on $\triangle P D$ than on $\triangle P F$, showing that increasing the budget limit allows the project duration to be extended, but that performance level increases up to a certain level and then remains almost unchanged. This is attributed to the features of the Cost vs Time linear curve and Progress vs Time exponential curve constructed for the CSFs, but it also reflects realistic ERP implementation. Additional budget is often reserved for more VS, more internal resources to the implementation team, or further training, in order to improve overall implementation performance (Umble et al., 2003). If extra budget does not bring any of the aforementioned resource, the ERP implementation performance will not be improved significantly once it reaches its maximum. Additional VS, training, and increased internal resources offer good opportunities for the teams who address CSFs to reach the performance thresholds quicker. This can lead to improved performance levels and increased chances of successful implementation. Guided by the results in Scenarios 1-4, the decision maker is recommended to make an appropriate estimate for the budget rather than overestimate it as the overestimated budget will not necessarily improve the performance level if it is only used to extend project duration. Extra budget is suggested to be spent on additional resources that can make contributions to improving the performance level.

According to Table 5, comparing Scenario 0 and Scenarios 1-4, as the budget limit is increased, and the time spent on CSFs increases, in the order of: PM (highest), TM, IT, Users and VS (lowest). This ranking is made by DSS_ERP taking into account the performance thresholds, progressing speeds and cost of the CSFs. If the objective is to maximise or achieve 
certain level of performance level, the CSFs are given greater priorities if they are associated with higher performance thresholds, lower progressing speeds and lower cost. The prioritised CSFs need to be allocated more resources (time) to make the requested contribution to ERP implementation.

The results in Table 5 indicate that the performance level achieved in Scenario 5 is down by 7.3\% in comparison with Scenario 1, although the implementation cost is the same and the project duration is increased by $8.7 \%$. Running this scenario helps the decision estimate the potential loss caused by the withdrawal of external vendor support, and to develop contingency plans for when it occurs. VS is essential to SMEs, as it can provide assistance in analysing the needs of the organisation, examining the organisation's readiness, on-site implementation assistance, regular system upgrade, and after sale and post implementation assistance. Most importantly, VS helps SMEs customise the ERP system to match the actual features of existing processes in the SMEs. Withdrawal of VS not only results in significant drops in the ERP implementation performance level, but also causes ERP implementation failure if the implementation team cannot take over the project successfully.

The results in Scenarios 6 and 7 once again highlight that PM is more important than Users, and should be given more focus. If the same extra budget is available, it is recommended to spend it on PM rather than Users. The magnitude of increased performance level is larger if the same level of extra budget and time is spent on PM instead of Users, which is attributed to PM having a higher performance threshold than Users. This finding is consistent with the findings in the analysis of goals 2 and 3. 


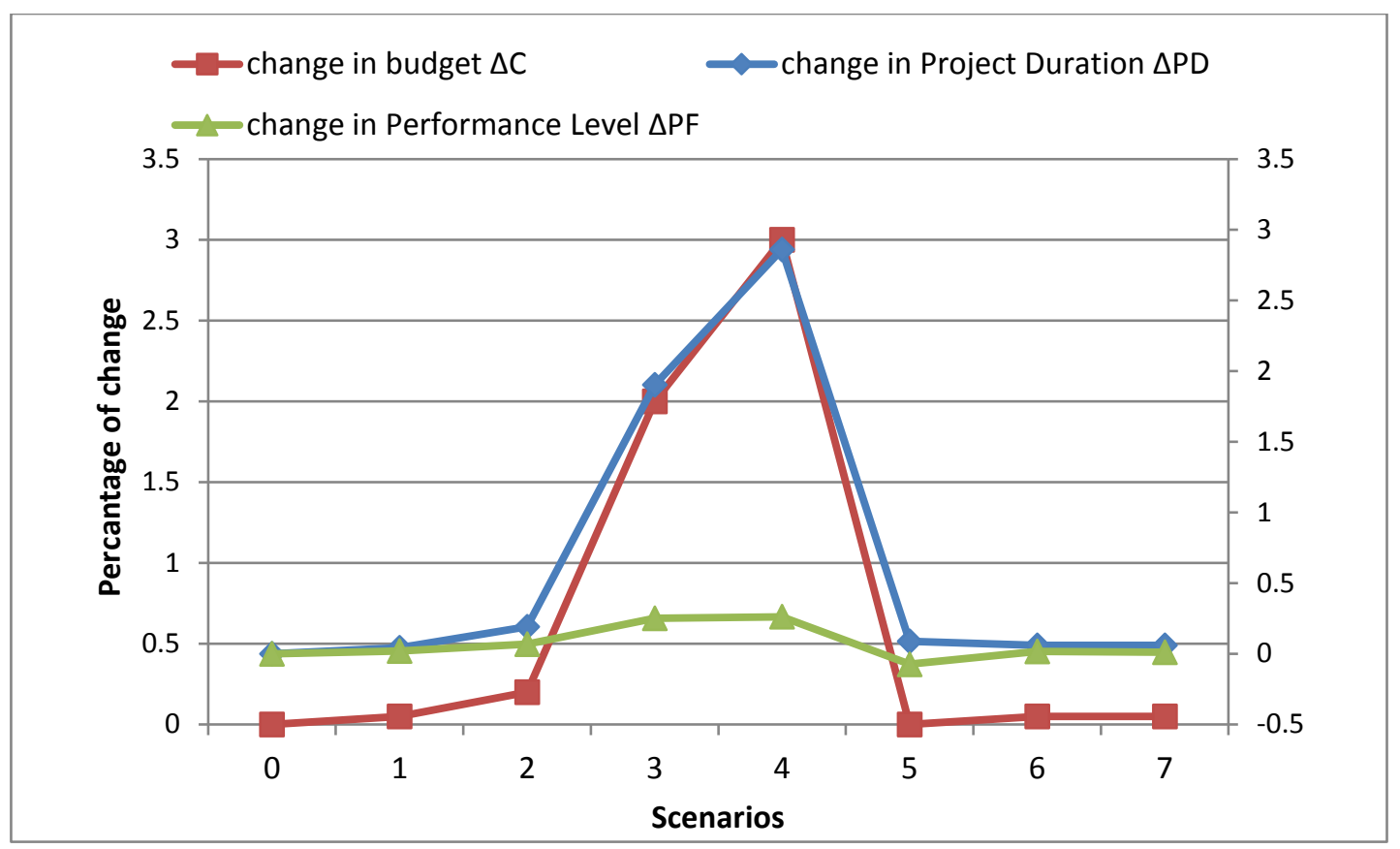

Fig. 4 A graphical comparison of What-If analysis

\section{Research synthesis}

ERP systems have enhanced and revolutionised the way organisations function, ultimately helping them become more productive and competitive. However, ERP implementation is a challenging, time consuming and expensive process, and can have adverse consequences if not well managed; the failure rate of ERP implementation has been estimated at between $60 \%$ and 90\% (Kwahk and Lee, 2008). Due to limited resources and a lack of perceived usefulness, ERP implementation becomes even more challenging to an SME. ERP implementation and optimisation have been investigated thoroughly, including study of such topics as ERP software selection, CSFs, business process reengineering, post-implementation and achievement of competitive advantage through ERP (Schlichter and Kraemmergaard, 2010). SMEs are recommended to focus on CSFs in order to improve the chances of successful implementation (Akkermans \& van Helden, 2002). However, the ERP implementation and optimisation literature lacks coverage of resource allocation to CSFs. Decision making tools that make it possible to predict required resources to address each CSF and to monitor the performance of each CSF and overall ERP project are not available in the literature. Without the ability to obtain more accurate estimates on required resources during the project planning phase, SMEs tend to underestimate based on inaccurate guesses and suffer project failures due to insufficient resources.

This research addresses the issues above and contributes to the undeveloped area by developing DSS_ERP using simulation and modelling approaches. Similar research has been 
conducted in Sun et al. (2005), where a simulation model is developed to help SMEs identify the key requirements to meet goals of ERP implementation. However, due to inadequate resources, the validity and generality of the simulation model developed by Sun et al. (2005) was limited as the observations were only on 6 case studies plus data generated using data fitting methods, and assumptions were made that discrepancies between observed data and the generated data were insignificant. The validity and generality are improved in this research by combining mathematical modelling with an empirical survey conducted on 60 SMEs. In addition, Sun et al's model (2005) model does not have the functionalities of predicting the resources needed for each CSF, and is of no use to SMEs in advanced planning.

Rouhani and Ravasan (2012) developed an expert system using an ANN method to predict the ERP success level based on the organisational profiles before system implementation. Although a comprehensive set of organisational factors are considered in this ANN trained system, and the system is validated with good predictive outcomes, the expert system does not provide a quantitative measure assessing the success level, or evaluate precisely how attention given to each factor during implementation impacts the success level. In comparison, DSS_ERP is a CSF based decision support system for ERP implementation, addressing five CSFs, and defining a quantitative evaluation of overall system success or performance level in terms of functionality achieved. DSS_ERP can help decision makers to determine the time and the budget on CSFs during ERP implementation, helping them to achieve their desired goals.

DSS_ERP combines analytical regression models, a simulation model and a nonlinear programming model. The analytical regression models are developed using realistic data, imitating the learning and performance of teams addressing CSFs along the time horizon. In contrast with Plaza and Rohlf (2008), where learning and performance of the project team is investigated, this research is more comprehensive and sophisticated: 1) five CSFs are addressed in our research in comparison with one CSF addressed in Plaza and Rohlf (2008); and 2) the analytical regression models developed are validated by statistical analysis using a simulation model, while the analytical model in Plaza and Rohlf (2008) is only tested in the context of a single organisation without verification.

The simulation model in this research provides dynamic views on the ERP implementation project processes, and is flexible enough to easily accommodate changes that represent specific circumstances in SMEs. With the simulation model, SMEs can try out decisions on total budget and project duration in a time compressed manner with no cost.

For the first time, a nonlinear programming model is developed to construct ERP implementation targets, and define limitations on budget and project duration as constraints. 
The model determines prioritisation of CSFs, and provides solutions on resource allocation, in such a way that predetermined targets are achieved.

The practical use and benefits of DSS_ERP are illustrated using Goal-Seeking and Whatif analysis. As demonstrated in Section 4, DSS_ERP serves as an analytical tool to monitor ERP implementation progresses along the time horizon, and it facilitates decision making process on resource allocations, to achieve predetermined implementation performance level, by calculating time and budget to be spent on each CSF. The What-if analysis demonstrates how potential risk or opportunities can be forecasted. Our research offers two practical contributions: (1) it offers guidance in resource acquisition and allocation that achieves predetermined ERP implementation performance level, within budget and time limits; and (2) it offers a risk analysis tool to analyse potential risk and opportunities caused by the changes to an ERP project, therefore helps SMEs to be better prepared and reduce failures.

\section{Conclusion}

This paper presents an integrated decision making system for ERP implementation, DSS_ERP, employing analytical regression models, a simulation model and a nonlinear programming model. The DSS_ERP uses the observed data obtained from empirical surveys to develop analytical regression models, which are verified by the simulation model before they are applied to construct the nonlinear programming model. The nonlinear programming models are employed to determine the resource allocations for the predetermined goals.

- ERP implementation is influenced by the CSFs addressed; the Cost vs Time and Progress vs Time curves obtained at CSF level illustrate how an ERP project performs against time, and can serve as a guidance tool for an SME considering ERP implementation.

- DSS_ERP can help decision makers to determine the priorities of CSFs through analysis of performance thresholds, progressing coefficients and cost. CSFs with higher progressing coefficients generate more rapid improvement during the early stages of the project, while CSFs with higher performance thresholds make greater contributions to the performance level in the later stages of the project.

- Taking the priorities into account, an SME can use DSS_ERP to allocate resources, defined as time spent on CSFs and their related progressing speeds, in such a way that the predetermined goals are met without breaching constraints. 
- DSS_ERP can also be used to analyse impacts on overall ERP performance of changes to resource allocations. This analysis helps decision makers to be better prepared for the potential risks and opportunities causey by changes, and to develop plans to meet the predetermined goals.

The results of this research are valid for the SMEs with 50-150 employees and have addressed the five CSFs in their ERP implementations: Project Management, Top Management, IT infrastructure, Users and Vendor Support.

- ERP implementation cost is increased along the time horizon, while performance level increases up to a point and remains unchanged;

- In terms of their impact on the ability to maximise or achieve a certain level of performance level within a budget limitation, CSFs are prioritised as: Project Management (highest), Top Management, IT infrastructure, Users and Vendor Support (lowest);

- SMEs are recommended to concentrate effort and resources on CSFs that have a greater impact on achieving their desired goals while optimising utilisation of resources. In Section 5.5, Scenarios about increasing progressing speeds of IT or budget on IT are not examined, considering that upgrading IT needs much greater budget and most SMEs cannot afford changing the existing IT infrastructure, but IT should be the subject of enhanced focus if the SMEs have the capability.

Future study and data collection are needed to identify how progressing coefficients of CSFs are influenced by staff allocation, staff training, and external consulting, and DSS_ERP will determine the resources needed to achieve the required progressing speeds, therefore facilitating ERP project planning and training strategy development. Further analysis will also be conducted to identify if and when to allocate more resources to CSFs with faster progressing speeds or higher performance levels. The DSS_ERP represented in this paper operates with the results of a survey of 60 SMEs, which results in the DSS_ERP being both generalisable and applicable. However, the methodology of developing DSS_ERP can work with results from any empirical study, and the analytical regression models, simulation model and nonlinear programming model can be revised accordingly. These features imply that the research is not restricted to ERP implementation, and future research will focus on real-world applications of the proposed decision support system for project management. 
Table 4 the solutions for goal-seeking analysis

\begin{tabular}{|c|c|c|c|c|c|c|c|c|c|c|c|c|c|c|c|}
\hline Goals & $\begin{array}{c}C \\
\text { (Dollars) }\end{array}$ & $\begin{array}{c}T \\
\text { (days) }\end{array}$ & $\begin{array}{c}\text { Performance level } \\
(\%)\end{array}$ & $\begin{array}{c}\text { Implementation cost } \\
\text { (Dollars) }\end{array}$ & $\begin{array}{c}\text { Project duration } \\
\text { (days) }\end{array}$ & $t_{1}$ & $t_{2}$ & $t_{3}$ & $t_{4}$ & $t_{5}$ & $k_{1}$ & $k_{2}$ & $k_{3}$ & $k_{4}$ & $k_{5}$ \\
\hline 1 & 100,000 & 180 & 73.276 & 100,000 & 115 & 33 & 17 & 39 & 17 & 9 & 0.045 & 0.163 & 0.040 & 0.076 & 0.143 \\
\hline 2 & 100,000 & 115 & 75.000 & 100,000 & 115 & 33 & 17 & 39 & 17 & 9 & 0.050 & 0.163 & 0.046 & 0.076 & 0.143 \\
\hline 3 & 100,000 & 115 & 75.000 & 100,000 & 115 & 33 & 17 & 39 & 17 & 9 & 0.045 & 0.199 & 0.040 & 0.076 & 0.189 \\
\hline
\end{tabular}

Table 5: the results of what-if analysis

\begin{tabular}{|c|c|c|c|c|c|c|c|c|c|c|c|}
\hline Scenarios & $\begin{array}{c}C \\
\text { (Dollar) }\end{array}$ & $\begin{array}{l}\Delta C \\
(\%)\end{array}$ & $\begin{array}{c}\text { Project duration } \\
\text { (days) }\end{array}$ & $\begin{array}{l}\triangle P D \\
(\%)\end{array}$ & $\begin{array}{c}\text { Performance level } \\
(\%)\end{array}$ & $\begin{array}{l}\Delta P F \\
(\%)\end{array}$ & $t_{1}$ & $t_{2}$ & $t_{3}$ & $t_{4}$ & $t_{5}$ \\
\hline 0 & 100,000 & 0 & 115 & 0 & 73.2760 & 0 & 33 & 17 & 39 & 17 & 9 \\
\hline 1 & 105,000 & 5 & 120 & 4.3 & 74.6685 & 1.9 & 35 & 17 & 40 & 18 & 10 \\
\hline 2 & 120,000 & 20 & 137 & 19.1 & 78.2747 & 6.8 & 40 & 19 & 46 & 21 & 11 \\
\hline 3 & 300,000 & 200 & 334 & 190 & 91.6626 & 25.1 & 100 & 35 & 113 & 56 & 30 \\
\hline 4 & 400,000 & 300 & 444 & 286 & 92.4204 & 26.1 & 133 & 44 & 150 & 76 & 19 \\
\hline 5 & 100,000 & 0 & 125 & 8.7 & 67.9063 & -7.3 & 40 & 18 & 46 & 21 & 0 \\
\hline 6 & 105,000 & 5 & 122 & 6.1 & 74.5208 & 1.7 & 40 & 17 & 39 & 17 & 9 \\
\hline 7 & 105,000 & 5 & 122 & 6.1 & 74.0339 & 1.0 & 33 & 24 & 39 & 17 & 9 \\
\hline
\end{tabular}




\section{References:}

1. Akkermans, H., and van Helden, K. (2002), "Vicious and virtuous cycles in ERP implementation: A case study of interrelations between critical success factors", European Journal of Information Systems, Vol. 11, pp. 35-46.

2. Babu A.J.G. and Suresh N. (1996), "Project management with time, cost, and quality considerations", European Journal of Operational Research, Vol.88, pp.320-327.

3. Bajwa D.S., Garcia, J.E. and Mooney, T. (2004), "An integrative framework for assimilation of enterprise resources planning systems; phases, antecedents, and outcome", Journal of Computer Information Systems, Vol.44, pp.81-90.

4. Balakrishnan N., Render B. and Stair R.M. (2007), "Managerial Decision Modeling with Spreadsheets", Prentice Hall, ISBN-10: 0131951149, ISBN-13: 9780131951143.

5. Bernroider E. (2008), "IT Governance for Enterprise Resource Planning supported with the DeLone-McLean Model of Information System Success", Information and Management, Vol. 45, No.5, pp.257-269.

6. Buonanno G. P., Pigni F. F., Ravarini A., Sciuto D., Tagliavini M. (2005), "Factors affecting ERP system adoption: a comparative analysis between SMEs and large companies", Journal of Enterprise Information Management, Vol.18, No.4, pp.384-426.

7. Cantu, R. (1999), “A framework for implementing enterprise resource planning systems in small manufacturing companies”, Master's Thesis, St. Mary's University, San Antonio.

8. Cebeci U. (2009), "Fuzzy AHP-based decision support system for selecting ERP systems in textile industry by using balanced scorecard", Expert Systems with Applications, Vol.36, No.5, pp.8900-8909.

9. Cioffi D.F. (2005), "A tool for managing projects: An analytic parameterization of the Scurve", International Journal of Project Management, Vol. 23, pp. 215 - 222.

10. Cumbie, B.A., Jourdan, Z., Peachey, T., Dugo, T.M., Craighead, C.W. (2005), "Enterprise resource planning research: Where are we now and where should we go from here", Journal of Information Technology: Theory and Application, Vol.7, No.2, pp.21-37.

11. El Sawah S., Tharwat, A.A. and Rasmy, M.H. (2008) "A quantitative model to predict the Egyptian ERP implementation success index", Business Process Management Journal, Vol.14, No.3, pp. 288-306.

12. Fulkerson D.R. (1961), “A network flow computation for project cost curves, Management Science”, Vol. 7, No. 2, pp.167-178.

13. Fox J. (2008), “Applied Regression Analysis and Generalized Linear Models", SAGE 
Publications, Second Edition, ISBN-10: 0761930426, ISBN-13: 978-0761930426.

14. Ho L.T. and Lin G.C.I. (2004), "Critical success factor framework for the implementation of integrated-enterprise systems in the manufacturing environment", International Journal of Production Research, Vol.42, Iss.17, pp.3731-3742.

15. Karsak E.E. and Ozogul C.O. (2009), “An integrated decision making approach for ERP system selection”, Expert Systems with Applications, Vol.36, No.1, pp.660-667.

16. King S.F. and Burgess T.F. (2006), "Beyond critical success factors: a dynamic model of enterprise system innovation", International Journal of Information Management, Vol. 26, pp. 59-69.

17. Koh Lenny S.C. and Simpson, M., (2005), "Changes and uncertainty in SME manufacturing environments using ERP”, Journal of Manufacturing Technology Management, Vol. 16, No. 6, pp. 629-653.

18. Kwahk, K.Y. and Lee, J.N. (2008), "The role of readiness for change in ERP implementation: theoretical bases and empirical validation", Information and Management, Vol. 45, No.7, pp. $474-481$.

19. Laukkanen S., Sarpola S. and Hallikainen P. (2007), "Enterprise size matters: objectives and constraints of ERP adoption", Journal of Enterprise Information Management, Vol.20, No.3, pp.319-334.

20. Lea B.R., Gupta M.C. and Yu W.B. (2005), “A prototype multi-agent ERP system: an integrated architecture and a conceptual framework", Technovation, Vol. 25, No.4, pp.433-441.

21. Loh T.C. and Koh S.C.L. (2004), "Critical elements for a successful enterprise resource planning implementation in small-and medium-sized enterprises", International Journal of Production Research, Vol.42, Iss.17, pp.3433-3455.

22. Markus M.L., Tanis, C. and Fenema, P.C. (2000), "Enterprise resource planning: multisite ERP implementation", Communication of the ACM, Vol.43, No. 4, pp.42-46.

23. Nah G.F.H., Lau, J.L.S. and Kuang. J. (2001) "Critical factors for successful integration of enterprise resource planning”, Business Process Management Journal, Vol.7, No.3, pp.285-296.

24. Parr A. and Shanks G. (2000), “A model of ERP project implementation”, Journal of Information Technology, Vol.15, pp. 289-303.

25. Plaza M. and Rohlf K. (2008), "Learning and performance in ERP implementation projects: a learning curve model for analyzing and managing consulting costs", International 
Journal of Production Economics, Vol.115, pp.72-85.

26. Project Management Institute Standards Committee (2000), A guide to the project management body of knowledge - 2000 ed. (PMBOK), Newtown Square, PA: Project Management Institute, Inc.

27. Raymond L. and Uwizeyemungu S. (2007), "A profile of ERP adoption in manufacturing SMEs", Journal of Enterprise Information Management, Vol. 20, No. 4, pp. 487 - 502.

28. Remus U. (2007), "Critical success factors for implementing enterprise portal: a comparison with ERP implementation", Business Process Management Journal, Vol.13, No.4, pp.538-552.

29. Rouhani S. and Ravasan A.Z. (2012), Scientia Iranica, Transactions E: Industrial Engineering, in press.

30. Schlichter B.R. and Kraemmergaard P. (2010), "A comprehensive literature review of the ERP research field over a decade", Journal of Enterprise Information Management, Vol.23, No.4, pp.486-520.

31. Somers T.M., \& Nelson, K. (2001), “The impact of critical success factors across the stages of enterprise resource planning implementation", Proceedings of the 34th Hawaii international conference on systems sciences (HICSS-34). January 3-6, Maui, Hawaii.

32. Stensrud E. (2001), "Alternative approaches to effort prediction of ERP projects", Information and Software Technology, Vol.43, No.7, pp.413-423.

33. Sun A.Y.T., Yazdani A. and Overend J. D. (2005), "Achievement assessment for enterprise resource planning (ERP) system implementations based on critical success factors (CSFs)", International Journal of Production Economics, Vol.98, pp.189-203.

34. Taha H.A. (2011), “Operations research: an introduction”, Prentice Hall, $9^{\text {th }}$ Edition, New Jersey, ISBN 10: 0-13-139199-2, ISBN 13: 978-0-13-139199-4.

35. Umble E.J., Haft R.R., and Umble M.M. (2003), "Enterprise resource planning: Implementation procedures and critical success factors", European Journal of Operational Research, Vol.146, pp.241-257.

36. Weiss N.A. (2012), "Introductory statistics", Addison-Wesley, 9th Edition, Boston, ISBN 13: 978-0-321-69122-4, ISBN-10: 0-321-69122-9.

37. Zach O. and Munkvold B.E., (2012), "Identifying reasons for ERP system customization in SMEs: a multiple case study", Journal of Enterprise Information Management, Vol. 25, No.5, pp. $462-478$. 
38. Zhang, Z., Lee, M.K.O., Huang, P., Zhang, L. and Huang, X. (2005), “A framework of ERP systems implementation success in China; an empirical study", International Journal of Production Economics, Vol.98, No.1, pp.56-80. 
Appendix A: Probability distributions of $t_{i}$

\begin{tabular}{|c|c|c|c|c|c|c|c|c|c|c|c|c|c|c|}
\hline$t_{1}$ & $n_{t_{1}}$ & $\operatorname{Prob}\left(t_{1}\right)$ & $t_{2}$ & $n_{t_{2}}$ & $\operatorname{Prob}\left(t_{2}\right)$ & $t_{3}$ & $n_{t_{3}}$ & $\operatorname{Prob}\left(t_{3}\right)$ & $t_{4}$ & $n_{t_{4}}$ & $\operatorname{Prob}\left(t_{4}\right)$ & $t_{5}$ & $n_{t_{5}}$ & $\operatorname{Prob}\left(t_{5}\right)$ \\
\hline 0 & 7 & 0.12 & 4 & 5 & 0.08 & 3 & 1 & 0.02 & 3 & 1 & 0.02 & 0 & 1 & 0.02 \\
\hline 1 & 1 & 0.02 & 12 & 4 & 0.06 & 7 & 1 & 0.02 & 4 & 1 & 0.02 & 3 & 1 & 0.02 \\
\hline 2 & 1 & 0.02 & 14 & 5 & 0.08 & 10 & 3 & 0.05 & 7 & 1 & 0.02 & 5 & 4 & 0.07 \\
\hline 4 & 2 & 0.03 & 17 & 3 & 0.05 & 14 & 5 & 0.08 & 10 & 4 & 0.07 & 6 & 5 & 0.08 \\
\hline 5 & 4 & 0.07 & 20 & 4 & 0.06 & 18 & 4 & 0.07 & 12 & 3 & 0.05 & 7 & 2 & 0.03 \\
\hline 7 & 8 & 0.13 & 21 & 9 & 0.15 & 20 & 7 & 0.12 & 14 & 5 & 0.08 & 9 & 3 & 0.05 \\
\hline 8 & 3 & 0.05 & 28 & 9 & 0.15 & 21 & 7 & 0.12 & 18 & 3 & 0.05 & 10 & 3 & 0.05 \\
\hline 9 & 1 & 0.02 & 30 & 4 & 0.06 & 25 & 3 & 0.05 & 20 & 2 & 0.03 & 11 & 3 & 0.05 \\
\hline 10 & 8 & 0.13 & 35 & 5 & 0.08 & 28 & 5 & 0.08 & 21 & 7 & 0.12 & 13 & 9 & 0.15 \\
\hline 12 & 2 & 0.03 & 40 & 3 & 0.05 & 30 & 8 & 0.13 & 24 & 2 & 0.03 & 15 & 2 & 0.03 \\
\hline 14 & 8 & 0.13 & 60 & 8 & 0.13 & 35 & 7 & 0.12 & 28 & 3 & 0.05 & 18 & 3 & 0.05 \\
\hline 18 & 2 & 0.03 & 77 & 2 & 0.03 & 40 & 3 & 0.05 & 30 & 4 & 0.07 & 20 & 5 & 0.08 \\
\hline 21 & 5 & 0.08 & 130 & 1 & 0.02 & 49 & 1 & 0.02 & 35 & 5 & 0.08 & 21 & 3 & 0.05 \\
\hline 28 & 1 & 0.02 & & & & 60 & 2 & 0.03 & 37 & 3 & 0.05 & 24 & 3 & 0.05 \\
\hline 30 & 5 & 0.08 & & & & 84 & 2 & 0.03 & 42 & 4 & 0.07 & 26 & 3 & 0.05 \\
\hline 45 & 1 & 0.02 & & & & 180 & 1 & 0.02 & 60 & 2 & 0.03 & 30 & 5 & 0.08 \\
\hline 84 & 1 & 0.02 & & & & & & & 63 & 2 & 0.03 & 33 & 4 & 0.07 \\
\hline & & & & & & & & & 70 & 2 & 0.03 & 44 & 1 & 0.02 \\
\hline & & & & & & & & & 84 & 2 & 0.03 & 84 & 1 & 0.02 \\
\hline & & & & & & & & & 100 & 2 & 0.03 & & & \\
\hline & & & & & & & & & 180 & 1 & 0.02 & & & \\
\hline
\end{tabular}

